\title{
Of Tilt and Twist
}

\author{
Zachary A. Holder, Richard C. Canfield, Rebecca A. McMullen \\ Department of Physics, Montana State University, Bozeman, MT \\ 59717-3840, U.S.A. \\ Robert F. Howard \\ National Solar Observatory/Kitt Peak ${ }^{1}$, Tucson, AZ 85726, U.S.A.
}

\begin{abstract}
Alexei A. Pevtsov
National Solar Observatory/Sacramento Peak 1 , PO Box 62, Sunspot, NM 88349, U.S.A.
\end{abstract}

\begin{abstract}
Using Mees Solar Observatory active-region vector magnetograms and Mt. Wilson Observatory full-disk longitudinal magnetograms, we measure both the twist and tilt of the magnetic fields of 368 active regions. This dataset clearly shows two well-known phenomena, Joy's law and the hemispheric helicity rule, as well as a lesser-known twist-tilt relationship, which is the point of this work. Those regions that closely follow Joy's law show no twist-tilt relationship, which is a predicted consequence of convective buffeting of initially untwisted and unwrithed flux tubes through the $\Sigma$ effect. Those regions that strongly depart from Joy's law show significantly larger than average twist and a very strong twist-tilt relationship. These properties suggest that the twist-tilt relationship in these regions is due to kinking of flux tubes that are highly twisted but not strongly writhed.
\end{abstract}

